\title{
Modification of claims-based measures improves identification of comorbidities in non-elderly women undergoing mastectomy for breast cancer: a retrospective cohort study
}

\author{
Katelin B. Nickel ${ }^{1}$, Anna E. Wallace², David K. Warren', Kelly E. Ball' ${ }^{1}$ Daniel Mines², Victoria J. Fraser ${ }^{1}$
} and Margaret A. Olsen ${ }^{1,3^{*}}$

\begin{abstract}
Background: Accurate identification of underlying health conditions is important to fully adjust for confounders in studies using insurer claims data. Our objective was to evaluate the ability of four modifications to a standard claims-based measure to estimate the prevalence of select comorbid conditions compared with national prevalence estimates.

Methods: In a cohort of 11,973 privately insured women aged 18-64 years with mastectomy from 1/04-12/11 in the HealthCore Integrated Research Database, we identified diabetes, hypertension, deficiency anemia, smoking, and obesity from inpatient and outpatient claims for the year prior to surgery using four different algorithms. The standard comorbidity measure was compared to revised algorithms which included outpatient medications for diabetes, hypertension and smoking; an expanded timeframe encompassing the mastectomy admission; and an adjusted time interval and number of required outpatient claims. A $\times 2$ test of proportions was used to compare prevalence estimates for 5 conditions in the mastectomy population to national health survey datasets (Behavioral Risk Factor Surveillance System and the National Health and Nutrition Examination Survey). Medical record review was conducted for a sample of women to validate the identification of smoking and obesity.

Results: Compared to the standard claims algorithm, use of the modified algorithms increased prevalence from 4. 79 to $6.79 \%$ for diabetes, 14.75 to $24.87 \%$ for hypertension, 4.23 to $6.65 \%$ for deficiency anemia, 1.78 to $12.87 \%$ for smoking, and 1.14 to $6.31 \%$ for obesity. The revised estimates were more similar, but not statistically equivalent, to nationally reported prevalence estimates. Medical record review revealed low sensitivity (17.86 \%) to capture obesity in the claims, moderate negative predictive value (NPV, $71.78 \%$ ) and high specificity (99.15\%) and positive predictive value (PPV, $90.91 \%)$; the claims algorithm for current smoking had relatively low sensitivity (62.50\%) and PPV (50.00 \%), but high specificity (92.19\%) and NPV (95.16\%).

(Continued on next page)
\end{abstract}

\footnotetext{
* Correspondence: molsen@wustl.edu

'Division of Infectious Diseases, Department of Medicine, Washington University School of Medicine, 660 South Euclid Ave. Campus Box 8051, St. Louis, MO 63110, USA

${ }^{3}$ Division of Public Health Sciences, Department of Surgery, Washington University School of Medicine, 660 South Euclid Ave. Campus Box 8100, St. Louis, MO 63110, USA

Full list of author information is available at the end of the article
} 
(Continued from previous page)

Conclusions: Modifications to a standard comorbidity measure resulted in prevalence estimates that were closer to expected estimates for non-elderly women than the standard measure. Adjustment of the standard claims algorithm to identify underlying comorbid conditions should be considered depending on the specific conditions and the patient population studied.

Keywords: Comorbidity, Administrative health claims data, Diabetes, Hypertension, Obesity, Mastectomy, Breast cancer

\section{Background}

Adjusting for comorbidities in observational studies is essential to account for underlying differences in populations under investigation. This is especially true when studying healthcare utilization, costs, and patient outcomes, in which underlying comorbid conditions are associated with the outcomes. The Charlson index [1], a widely-used measure of 19 comorbidities, was adapted for administrative data using ICD-9-CM (International Classification of Diseases, $9^{\text {th }}$ Revision, Clinical Modification) codes [2,3]. A second commonly used comorbidity measure with claims data developed by Elixhauser includes 29 medical conditions [4, 5]. These measures were developed and validated in hospitalized patients, and therefore may be more applicable to older, sicker populations.

A number of studies have compared the performance of the Charlson and Elixhauser measures [6-12]. The best measure has generally been determined based on improvement in explanatory power with regard to a specific outcome, usually mortality. Many studies have concluded that that these measures perform equally well [7, 9-11], while others have found the Elixhauser classification improved prediction of in-hospital $[8,12]$ and longerterm mortality $[6,12]$. Several investigators have expanded the parameters of these two comorbidity measures to include physician [10, 13-15], outpatient, and auxiliary claims $[10,14,15]$ and different lookback periods relative to the index event (e.g., one or two years of prior data and/or including the index admission) [10, 12, 14, 15].

We have an ongoing study of risk factors for postoperative complications in women under the age of 65 years after mastectomy and breast reconstruction using commercial insurer claims data $[16,17]$. Breast reconstruction is most commonly performed in younger women [18] who have fewer underlying medical conditions (M. Olsen, unpublished results, National Inpatient Data) and lower annual healthcare costs than elderly women [19], suggesting less healthcare utilization. Fewer healthcare encounters and hospitalizations could limit the identification of comorbid conditions in claims data. For example, methods that perform well in elderly populations, e.g., using only diagnoses on inpatient hospital claims, may not be optimal for younger, generally healthy populations with infrequent hospitalizations.

To address some of the challenges of capturing comorbidities in younger populations, we selected diabetes, hypertension, anemia (including iron, folate, vitamin B12, protein, and other nutritional deficiency anemias), smoking, and obesity as representative examples of commonly occurring underlying health conditions in younger persons, and to illustrate the impact of modifications to the standard claims algorithm to improve capture of comorbidities. One alteration included the addition of diagnosis codes during the index hospital admission, to capture conditions that might be more commonly coded during a hospitalization than during prior outpatient encounters. All five conditions we included in our study pertain to this example, since they may be considered relevant to care provided during an inpatient hospitalization, but may not be relevant to service(s) or procedure(s) rendered during an outpatient visit, a requirement for health insurance coding [20]. Another alteration included allowing only a single diagnosis code to identify conditions for which testing is not necessary, such as obesity and smoking. A third alteration consisted of relaxing the requirement for spacing of diagnoses coded during outpatient encounters at least 30 days apart, because of the potential for clustering of care within a short time frame in younger women diagnosed with breast cancer. We also examined the effect of prescription drug claims to identify a condition, using hypertension and diabetes as relevant conditions. Our objective was to compare the impact of these modifications to the standard claims algorithm to identify a comorbid health condition, using the expected population prevalence of the comorbid health condition and medical record review to signal improvement.

\section{Methods}

\section{Data source}

We conducted a retrospective cohort study using the HealthCore Integrated Research Database $\left(\right.$ HIRD $\left.^{\text {SM }}\right)$. We used data in the HIRD ${ }^{\mathrm{SM}}$ from individuals enrolled in 12 Anthem-affiliated plans for this study. Anthem is an independent licensee of the Blue Cross and Blue Shield Association and serves its members as the Blue Cross licensee (California), and the Blue Cross and Blue 
Shield licensee (Colorado, Connecticut, Georgia, Indiana, Kentucky, Maine, Missouri (excluding 30 counties in the Kansas City area), Nevada, New Hampshire, New York, Ohio, Virginia (excluding the Northern Virginia suburbs of Washington, DC), and Wisconsin). Data in the HIRD ${ }^{\mathrm{SM}}$ include all fully-adjudicated claims submitted for reimbursement from providers, facilities, and outpatient pharmacies linked to health plan enrollment information.

Fully insured women with health plan enrollment that included non-capitated medical coverage of hospital and physician services and prescription drug coverage were eligible for inclusion in the study cohort. Men were excluded due to the rare incidence of breast cancer in men. Women lacking continuous coverage from 365 days before through 30 days after mastectomy were excluded since comorbid conditions could not be fully measured. Additional exclusions included women with diagnosis codes for end-stage renal disease because of potentially incomplete claims, ICD-9-CM diagnosis code or prescription claim suggesting HIV positive status at any time for privacy considerations, and organ transplant in the year before mastectomy due to the rare nature of their underlying illness. Claims were restricted to paid claims.

The study data contained up to 5 ICD-9-CM diagnosis codes and 5 ICD-9-CM procedure codes per claim among women with a mastectomy in 20042008 and up to 12 ICD-9-CM diagnosis codes and 8 ICD-9-CM procedure codes among women with a mastectomy in 2009-2011. Inpatient facility claims also included Uniform Billing (UB-04) revenue and Healthcare Common Procedure Coding System (HCPCS) codes, while outpatient facility and provider claims included Current Procedural Terminology, $4^{\text {th }}$ edition (CPT-4) and HCPCS codes. Pharmacy claims contained National Drug Codes, which were linked to Generic Product Identifier codes to identify medications and drug groups.

\section{Patient population}

We identified mastectomy operations among women aged 18-64 years from 1/1/2004-12/31/2011 using ICD-9-CM procedure codes $85.41-85.48$ from inpatient facility claims and/or CPT-4 procedure codes 19180, 19200-19240, 19303, and 19305-19307 from outpatient facility and provider claims. We included only the first mastectomy per woman during the time period. The patient population was further refined by excluding operations coded by a provider- or facility-only without additional evidence for operation (i.e., anesthesia, pathology, or surgery revenue code) and mastectomy with codes for breast-conserving surgery if provider and other claims suggested breastconserving surgery was more likely to increase the chance that a mastectomy was performed [17].

\section{Comorbidity algorithms}

We used the ICD-9-CM diagnosis codes from the Elixhauser classification [4] to define diabetes, hypertension, anemia, and obesity (see Additional file 1). Since smoking is not included in the Elixhauser list of comorbidities; we used ICD-9-CM diagnosis codes for history of tobacco use and tobacco disorder to define smoking. We did not restrict the identification of conditions by diagnosis-related group [4] since we wanted to identify all relevant comorbid conditions at the time of mastectomy. We examined the impact of outpatient prescription claims for medications used to treat diabetes, hypertension, and smoking cessation to enhance the detection of these conditions (see Additional file 1). In order to focus on diagnoses recorded by clinicians, we excluded provider and outpatient facility claims containing only CPT-4 or UB-04 revenue codes for pharmacy, diagnostic radiology/cardiology/pulmonology, clinical laboratory, physical/occupational therapy, speech pathology or ambulance services. The timeframe used to identify comorbid conditions, frequency and interval between outpatient and provider claims, and use of prescription drug claims were changed sequentially to determine how the changes impacted the final prevalence calculated for each underlying condition (Table 1).

For the standard claims algorithm (Algorithm 1), we used medical claims from -365 through -1 days before mastectomy and required $\geq 2$ provider or outpatient facility claims spaced $>30$ days apart or $\geq 1$ inpatient claim to identify comorbid conditions, as described by Klabunde [13]. For algorithm 2, we expanded the timeframe to capture comorbid conditions in medical claims through 7 days after mastectomy. While the standard claims algorithm described by Klabunde does not include identification of comorbid conditions during the hospital admission because some conditions could have new onset during the hospital admission (e.g., blood loss anemia, electrolyte disturbances), the comorbid conditions we selected for these analyses were most likely pre-existing at the time of mastectomy. Additionally, our study population consisted of younger, privately insured women with a low frequency of inpatient hospitalizations in the year prior to mastectomy, giving little opportunity to identify comorbid conditions in prior inpatient admissions. We suspected that some health conditions would be more likely to be coded during the mastectomy hospitalization (e.g., smoking) because of its relevance to surgery, so this provided additional motivation to determine the impact of adding the surgical hospitalization to the algorithm to identify underlying health conditions.

The frequency and interval requirements for provider/ outpatient facility claims were altered in algorithm 3. Because of potential clustering of medical encounters in women with a diagnosis of breast cancer, we considered $\geq$ 
Table 1 Parameters for each algorithm used to identify comorbidities

\begin{tabular}{|c|c|c|c|c|c|}
\hline Algorithm & Comorbidity & $\begin{array}{l}\text { Timeframe relative } \\
\text { to mastectomy date } \\
\text { (in days) }\end{array}$ & $\begin{array}{l}\text { Number of inpatient } \\
\text { facility medical claims } \\
\text { required }\end{array}$ & $\begin{array}{l}\text { Interval and number of } \\
\text { provider or outpatient } \\
\text { facility medical claims required }\end{array}$ & $\begin{array}{l}\text { Number of prescription } \\
\text { drug }(R x) \text { claims } \\
\text { required }\end{array}$ \\
\hline Algorithm 1 & $\begin{array}{l}\text { Diabetes, hypertension, } \\
\text { deficiency anemia, smoking, } \\
\text { obesity }\end{array}$ & -365 to -1 & $1+$ & $2+$ more than 30 days apart & $\mathrm{n} / \mathrm{a}$ \\
\hline Algorithm 2 & $\begin{array}{l}\text { Diabetes, hypertension, } \\
\text { deficiency anemia, smoking, } \\
\text { obesity }\end{array}$ & -365 to +7 & $1+$ & $2+$ more than 30 days apart & $\mathrm{n} / \mathrm{a}$ \\
\hline \multirow[t]{2}{*}{ Algorithm 3} & $\begin{array}{l}\text { Diabetes, hypertension, } \\
\text { deficiency anemia }\end{array}$ & -365 to +7 & $1+$ & $2+$ & $\mathrm{n} / \mathrm{a}$ \\
\hline & Smoking, obesity & -365 to +7 & $1+$ & $1+$ & $\mathrm{n} / \mathrm{a}$ \\
\hline \multirow[t]{3}{*}{ Algorithm 4} & Diabetes & $\begin{array}{l}-365 \text { to }+7 \\
-365 \text { to }-1 \mathrm{Rx}\end{array}$ & $1+$ & $2+$ & $1+$ \\
\hline & Hypertension & $\begin{array}{l}-365 \text { to }+7 \\
-365 \text { to }-1 \mathrm{Rx}\end{array}$ & $1+$ & $2+$ & $\begin{array}{l}1+\text { Rx plus } 1 \\
\text { hypertension medical } \\
\text { claim }\end{array}$ \\
\hline & Smoking & $\begin{array}{l}-365 \text { to }+7 \\
-365 \text { to }-1 R x\end{array}$ & $1+$ & $1+$ & $1+$ \\
\hline
\end{tabular}

n/a not applicable

2 provider/outpatient facility claims consistent with the diagnosis of interest (i.e., dropped the requirement for spacing of outpatient diagnoses $>30$ days apart). For smoking and obesity, we considered single provider/outpatient facility claim(s) sufficient evidence since these conditions do not require diagnostic workup.

One or more prescription claims for medications to treat diabetes (oral hypoglycemic, insulin), hypertension, and smoking cessation from -365 to -1 days before mastectomy were added to algorithm 4 to detect these conditions (see Additional file 1). At least one medical claim with a hypertension diagnosis was required in addition to a prescription claim for an anti-hypertensive medication, since drugs used for hypertension may also be used to treat other conditions.

\section{Comparison populations}

Survey results from the 2007 Behavioral Risk Factor Surveillance System (BRFSS) [21] and 2000 National Health and Nutrition Examination Survey (NHANES) [22] were used for the national estimates of the prevalence for each of the comorbid conditions. To best approximate our privately insured mastectomy cohort, weighted estimates for diabetes, hypertension, smoking, and obesity were calculated from female BRFSS respondents aged 18-64 years old with health insurance. In the surveys diabetes and hypertension were captured by the question "Have you ever been told by a doctor that you have (diabetes/high blood pressure)?” In both surveys BMI was defined using reported height and weight, and current smoking was defined on the basis of two questions: "Have you smoked at least 100 cigarettes in your entire life?" and "Do you now smoke cigarettes every day, some days, or not at all?" The prevalence of iron deficiency anemia (used as a proxy for all nutritional deficiency anemia) among adult females was available in a publication referencing NHANES data [22]. In the NHANES survey iron deficiency anemia was defined based on laboratory results from the respondent's blood sample. The age group level prevalence estimates from the NHANES publication were averaged for 20-64 year olds to establish a single prevalence estimate for deficiency anemia. Because obesity is a risk factor for breast cancer [23], and hypertension and diabetes are more prevalent in obese persons, we assumed that women undergoing mastectomy would have higher rates of the selected conditions than the survey populations. While the survey populations may not be a gold-standard, the prevalence rates should serve as a baseline threshold for comparison to our study population.

For additional comparisons, a subset of the privately insured mastectomy population with postoperative ICD9-CM diagnosis code(s) suggestive of wound complications was selected for medical record review. We selected this subset in order to validate the ICD-9-CM diagnosis codes for wound complications (manuscript in preparation), in addition to validation of the codes for obesity and smoking. For this subset height, weight, and current and past smoking history were abstracted from the medical records. Body mass index $(\mathrm{BMI}) \geq 30$ was used to define obesity.

\section{Analysis}

Wald confidence intervals were calculated for each condition prevalence estimate. A chi-square test of proportions was used to compare the different algorithms in 
the mastectomy cohort and estimates from BRFSS and NHANES. Sensitivity, specificity, positive predictive value (PPV), and negative predictive value (NPV) were calculated to compare smoking and obesity data from the claims to the medical record results. All data management and statistical analyses were performed using SAS v9.3 (SAS Institute Inc., Cary, NC). This study was approved by the Human Research Protection Office at Washington University and by the Quorum Review IRB for the research activity at HealthCore.

\section{Results}

The mastectomy cohort included 11,973 women aged 1864 years with at least one year of health insurance enrollment prior to mastectomy from 1/1/2004-12/31/2011. The average age of women in the population was 51 years, and all 4 regions of the U.S. were represented in the cohort (Table 2). More than $60 \%$ of women underwent mastectomy for locally invasive breast cancer, and $59 \%$ of women had immediate reconstruction at the time of mastectomy, primarily involving an implant.

For all five conditions, each successive change to the comorbidity algorithm resulted in an increase in

Table 2 Characteristics of 11,973 women in the mastectomy population

\begin{tabular}{ll}
\hline Characteristic & $\mathrm{n}(\%)$ \\
\hline Age, mean (standard deviation) & $50.79(8.36)$ \\
Region & \\
$\quad$ Northeast & $2,122(17.72)$ \\
South & $3,966(33.12)$ \\
Midwest & $2,455(20.50)$ \\
West & $3,403(28.42)$ \\
Unknown & $27(0.23)$ \\
Surgical procedure & \\
Mastectomy-only & $4,887(40.82)$ \\
Mastectomy with immediate & $5,440(45.44)$ \\
implant reconstruction & $1,228(10.26)$ \\
Mastectomy with immediate & \\
flap reconstruction & $418(3.49)$ \\
Mastectomy with immediate & \\
flap plus implant reconstruction & $4,995(41.72)$ \\
Bilateral mastectomy & \\
Indication for mastectomy & \\
Metastatic cancer & $298(2.49)$ \\
Regional cancer & $2,385(19.92)$ \\
Local breast cancer & $7,381(61.65)$ \\
Carcinoma in situ & $1,529(12.77)$ \\
Prophylactic & $308(2.57)$ \\
Benign/other & \\
\hline
\end{tabular}

prevalence of the condition (Fig. 1). Compared to the standard claims algorithm (algorithm 1), the revised prevalence estimates increased from $4.79 \%$ (95\% confidence interval $[\mathrm{CI}] 4.40,5.17)$ to $6.79 \%$ (95\% CI 6.34, 7.24) for diabetes, $14.75 \%$ (95\% CI 14.11, 15.39) to $24.87 \%$ (95 \% CI 24.10, 25.65) for hypertension, $4.23 \%$ (95\% CI 3.87, 4.60) to $6.65 \%$ (95 \% CI 6.20, 7.09) for anemia, 1.78 \% (95 \% CI 1.54, 2.02) to $12.87 \%$ (95 \% CI 12.27, 13.47) for smoking, and $1.14 \%(95 \%$ CI 0.95 , 1.33 ) to $6.31 \%$ (95 \% CI $5.88,6.75)$ for obesity.

\section{Impact of successive changes to comorbidity algorithm}

The impact of each successive change to the algorithm varied by comorbid condition (Fig. 1). When the timeframe was expanded to include diagnoses coded during the surgical admission (algorithm 2), the percentage of women with diabetes, hypertension, anemia, smoking, and obesity increased 19, 37, 26, 319, and $196 \%$, respectively. The impact of dropping the requirement for spacing of provider/outpatient claims $>30$ days apart in algorithm 3 resulted in an additional $5 \%$ increase in diabetes, $8 \%$ for hypertension, and $25 \%$ for anemia. Requiring only a single provider/outpatient claim for smoking and obesity in algorithm 3 resulted in increased prevalence of smoking (70 \%) and obesity (88 \%) compared to algorithm 2. Finally, the addition of prescription claims in algorithm 4 increased the percentage of women with diabetes by $13 \%$, hypertension by $14 \%$, and smoking by $2 \%$ (Fig. 1). Each successive change from one algorithm to the next resulted in a statistically significant increase in the comorbid condition prevalence with two exceptions (algorithm 4 and smoking, algorithm 3 and diabetes).

We conducted a sensitivity analysis to see if the performance of the algorithms to identify comorbid health conditions varied by age (Table 3 ). We found the greatest overall percent improvement compared to algorithm 1 in the youngest age group (18-47 years) for diabetes, hypertension, and obesity, while improvement was greatest for deficiency anemia and smoking in the middle age group (48-55 years).

In the final algorithm for each comorbid condition (i.e., algorithm 3 for anemia and obesity, algorithm 4 for diabetes, hypertension, and smoking), provider/outpatient facility claims contributed more to the prevalence estimate than inpatient facility claims (Table 4). Prescription drug claims captured more women with diabetes than either inpatient facility or outpatient facility/provider claims alone. For hypertension, prescription claims plus at least a single coded medical claim identified more women than were identified by either a single inpatient facility or $\geq 2$ outpatient facility/provider claims. In contrast, smoking cessation 


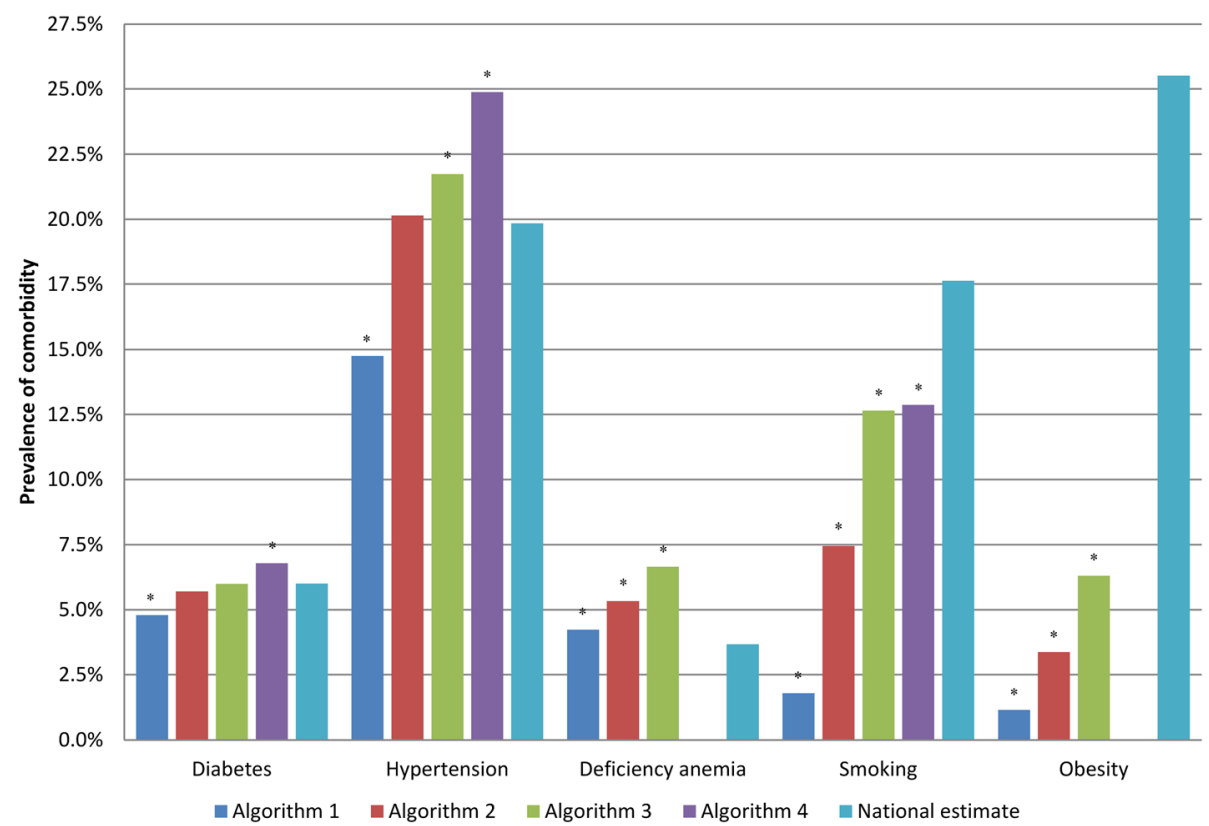

Fig. 1 Comparison of Prevalence Estimates for Comorbidities by Algorithm Compared to National Estimates. ${ }^{*}$ Significantly different from national estimate

Table 3 Comparison of prevalence estimates for comorbidities by age tertile and improvement by algorithm

\begin{tabular}{|c|c|c|c|c|c|c|c|}
\hline \multirow[t]{2}{*}{ Comorbidity } & \multirow[t]{2}{*}{ Algorithm $^{a}$} & \multicolumn{2}{|c|}{ Age $18-47$ years } & \multicolumn{2}{|c|}{ Age $48-55$ years } & \multicolumn{2}{|c|}{ Age $56-64$ years } \\
\hline & & $\begin{array}{l}\text { Prevalence, } \\
\%\end{array}$ & $\begin{array}{l}\text { Improvement compared } \\
\text { to algorithm 1, \% }\end{array}$ & $\begin{array}{l}\text { Prevalence, } \\
\%\end{array}$ & $\begin{array}{l}\text { Improvement compared } \\
\text { to algorithm 1,\% }\end{array}$ & $\begin{array}{l}\text { Prevalence, } \\
\%\end{array}$ & $\begin{array}{l}\text { Improvement compared } \\
\text { to algorithm 1, \% }\end{array}$ \\
\hline \multirow[t]{4}{*}{ Diabetes } & 1 & 1.39 & & 4.56 & & 8.57 & \\
\hline & 2 & 1.92 & 38.13 & 5.26 & 15.35 & 10.11 & 17.97 \\
\hline & 3 & 2.09 & 50.36 & 5.62 & 23.25 & 10.46 & 22.05 \\
\hline & 4 & 2.64 & 89.93 & 6.35 & 39.25 & 11.57 & 35.01 \\
\hline \multirow[t]{4}{*}{ Hypertension } & 1 & 5.62 & & 14.14 & & 24.93 & \\
\hline & 2 & 8.45 & 50.36 & 19.16 & 35.50 & 33.38 & 33.89 \\
\hline & 3 & 9.24 & 64.41 & 20.98 & 48.37 & 35.59 & 42.76 \\
\hline & 4 & 11.04 & 96.44 & 24.00 & 69.73 & 40.23 & 61.37 \\
\hline \multirow{3}{*}{$\begin{array}{l}\text { Deficiency } \\
\text { anemia }\end{array}$} & 1 & 4.44 & & 4.35 & & 3.67 & \\
\hline & 2 & 5.47 & 23.20 & 5.86 & 34.71 & 4.66 & 19.18 \\
\hline & 3 & 6.77 & 52.48 & 7.37 & 69.43 & 5.82 & 48.85 \\
\hline \multirow[t]{4}{*}{ Smoking } & 1 & 1.78 & & 1.61 & & 1.94 & \\
\hline & 2 & 7.03 & 294.94 & 7.94 & 393.17 & 7.41 & 281.96 \\
\hline & 3 & 11.96 & 571.91 & 13.51 & 739.13 & 12.55 & 546.91 \\
\hline & 4 & 12.20 & 585.39 & 13.77 & 755.28 & 12.70 & 554.64 \\
\hline \multirow[t]{3}{*}{ Obesity } & 1 & 0.84 & & 1.28 & & 1.31 & \\
\hline & 2 & 2.79 & 232.14 & 3.49 & 172.66 & 3.86 & 194.66 \\
\hline & 3 & 5.07 & 503.57 & 6.20 & 384.38 & 7.74 & 490.84 \\
\hline
\end{tabular}


Table 4 Prevalence of comorbidities by claims data source in the final algorithm ${ }^{\mathrm{a}}$ for 11,973 women

\begin{tabular}{|c|c|c|c|c|c|}
\hline Claim source & $\begin{array}{l}\text { Diabetes, } \\
\mathrm{n}(\%)\end{array}$ & $\begin{array}{l}\text { Hypertension, } \\
\mathrm{n}(\%)\end{array}$ & $\begin{array}{l}\text { Deficiency anemia, } \\
\mathrm{n}(\%)\end{array}$ & $\begin{array}{l}\text { Smoking, } \\
\mathrm{n}(\%)\end{array}$ & $\begin{array}{l}\text { Obesity, } \\
\mathrm{n}(\%)\end{array}$ \\
\hline Inpatient facility & $366(3.06)$ & $1,382(11.54)$ & $215(1.80)$ & $746(6.23)$ & $314(2.62)$ \\
\hline Provider or outpatient facility & $637(5.32)$ & $2,147(17.93)$ & $642(5.36)$ & $1,017(8.49)$ & $540(4.51)$ \\
\hline Prescription drug & $641(5.35)$ & $2,577(21.52)^{b}$ & $\mathrm{n} / \mathrm{a}$ & $61(0.51)$ & $\mathrm{n} / \mathrm{a}$ \\
\hline $\begin{array}{l}\text { Inpatient facility, provider, or outpatient } \\
\text { facility (algorithm 3) }\end{array}$ & $718(6.00)$ & $2,603(21.74)$ & $796(6.65)$ & $1,515(12.65)$ & $756(6.31)$ \\
\hline $\begin{array}{l}\text { Inpatient facility, provider, outpatient facility, } \\
\text { or prescription drug (algorithm 4) }\end{array}$ & $813(6.79)$ & $2,978(24.87)^{b}$ & $\mathrm{n} / \mathrm{a}$ & $1,541(12.87)$ & $\mathrm{n} / \mathrm{a}$ \\
\hline
\end{tabular}

n/a not applicable

a Final algorithm was algorithm 3 for deficiency anemia and obesity and algorithm 4 for diabetes, hypertension, and smoking; see Table 1 for description of each algorithm

${ }^{\mathrm{b}}$ By definition all with $\mathrm{Rx}$ had at least one facility or provider medical claim

prescription claims did not add significantly to the prevalence estimate of smoking (Table 4).

We examined the combination of data sources coded for individual conditions and relevant medications (Fig. 2). In algorithm $4,32 \%$ of women who met the definition for diabetes and $34 \%$ of women with hypertension had both provider/outpatient facility claims and medication claims for the condition. Thirty percent of women with diabetes and $29 \%$ with hypertension were coded positive in all three data sources (i.e., inpatient facility, provider/outpatient facility, and prescription drug). Provider/outpatient facility claims alone were the most common source coded for anemia (73\%), smoking (49\%), and obesity (58\%), followed by coding on inpatient facility claims alone with 19,32 , and $29 \%$ respectively. Only a small percentage had both inpatient facility and provider/outpatient facility claims positive for anemia, smoking, or obesity $(8,16$, and $13 \%$, respectively).

To determine the clustering of coding of diagnoses before mastectomy in this younger population, we analyzed the diagnosis codes present on medical claims in the month prior to mastectomy. In this time period, $64 \%$ of the diagnoses on medical claims related to the diagnosis of breast cancer (invasive or in situ cancer, history of breast cancer) or suspicion of cancer (e.g., mammographic abnormality, lump in breast). Invasive or in situ cancer codes were particularly common, accounting for $44 \%$ of all diagnoses on medical claims in the month prior to mastectomy.

\section{Prevalence of comorbid conditions in claims data versus national estimates}

We calculated national estimates for the prevalence of diabetes (6.01\%), hypertension (19.84\%), smoking $(17.63 \%)$ and obesity (25.51 \%) in adult insured women under the age of 65 years using data from the 2007 BRFSS, and used the estimate of iron deficiency anemia (3.67\%) derived from the 2000 NHANES [22]. The revised estimate of diabetes prevalence in our mastectomy population calculated by algorithms $2(5.70 \% ; p=0.160)$ and $3(6.00 \% ; p=0.952)$ and the estimate of hypertension prevalence calculated in algorithm $2(20.15 \% ; p=$ 0.402 ) were not significantly different from the national estimates (Fig. 1).

\section{Comorbid condition prevalence in claims data by year and compared to medical record review}

We compared the prevalence of the five comorbid conditions among women with mastectomy in 2004-2008 versus 2009-2011 to look at the impact of the increase in ICD-9-CM diagnosis code fields in the HIRD ${ }^{\mathrm{SM}}$ from 5 to 12 (see Additional file 1). For diabetes, hypertension, smoking, and obesity, the prevalence was significantly higher in 2009-2011 for all 4 algorithms compared to the earlier time period. The prevalence of anemia was higher among women with mastectomy in 2004-2008, although it did not reach statistical significance for algorithm 2 .

We abstracted information on current smoking and height and weight to calculate BMI from the medical records of 290 women in the cohort, although not all records contained the specific documentation $(75 \%$ had information on smoking and $60 \%$ of records had both weight and height). The specificity of coding for both current and ever-smoking and for obesity was very high (Table 5). The PPV of coding was high for both conditions, although the PPV for coding was lower when smoking was defined as current smoking. Conversely, the sensitivity and NPV of coding for smoking were higher when coded smoking was compared to current smoking status documented in the medical record, rather than ever smoked. For obesity, the specificity and PPV were high and the sensitivity and NPV were low for all 3 algorithms (Table 5). The BMI for obese women who were coded with ICD-9-CM diagnosis codes for obesity in claims was significantly higher than the BMI in obese women with no encounters coded for obesity (median BMI 40.20 vs. $32.85, p<0.001$, Mann-Whitney U test). 


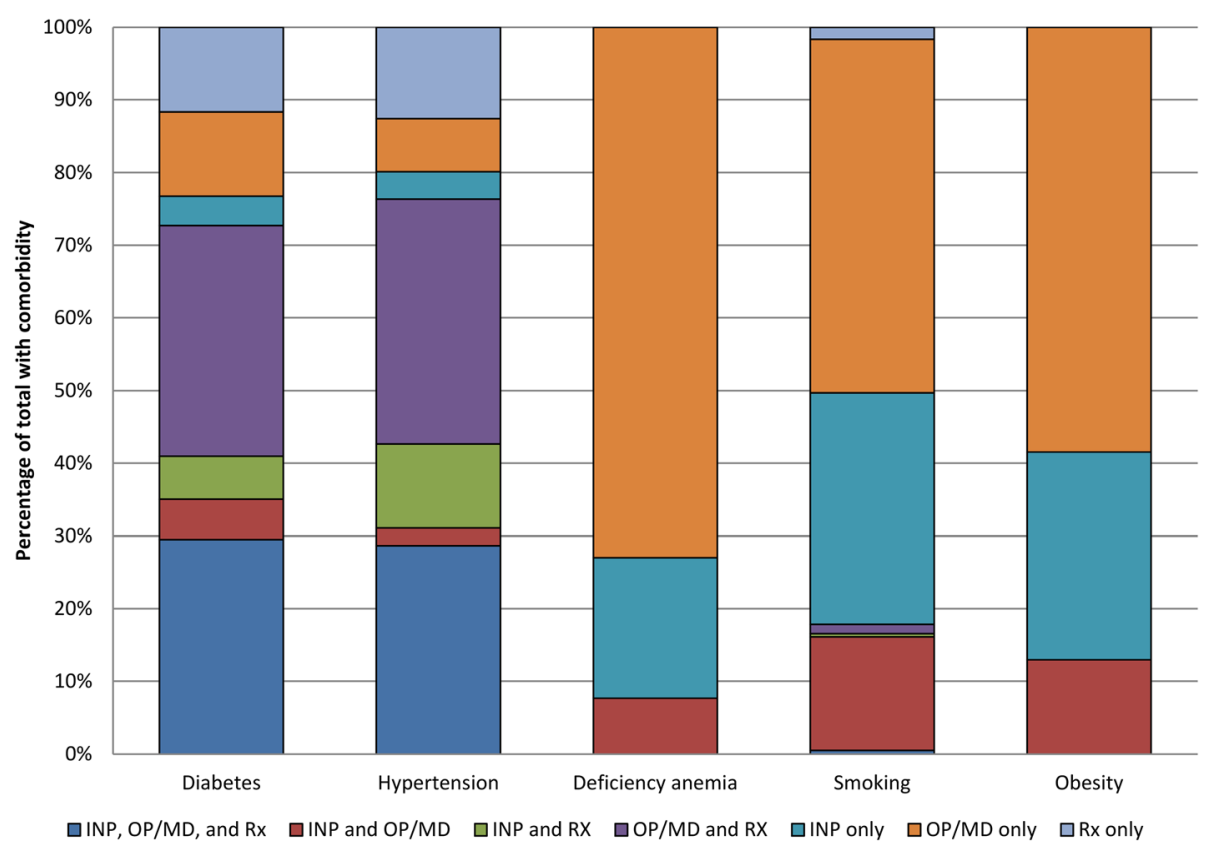

Fig. 2 Claim Sources Used to Define Comorbidities in Final Algorithm* Among Those Positive for the Comorbidity. INP=inpatient facility claim, $\mathrm{OP} / \mathrm{MD}=$ outpatient facility or provider claim, $\mathrm{Rx}=$ prescription drug claim. *algorithm 3 for deficiency anemia and obesity, algorithm 4 for diabetes, hypertension, and smoking

\section{Discussion}

We found progressively increasing prevalence of comorbid conditions in a population of younger women who underwent mastectomy by including the surgical admission to identify conditions, eliminating minimum spacing or need for more than one outpatient/provider claims coded for the condition, and identifying conditions with prescription claims, where possible. For all comorbid conditions, each change to the algorithm resulted in additional women detected with the underlying health condition and prevalence values closer to the national estimates for insured adult women, particularly for the younger women in our population. These five comorbid conditions served as illustrative examples

Table 5 Results of chart validation of smoking and obesity compared with insurance claims

$\begin{array}{llllllll}\text { Chart and claims, } \mathrm{n} & \text { Chart only, } \mathrm{n} & \text { Claims only, } \mathrm{n} & \text { Not in chart or claims, } \mathrm{n} & \text { Sensitivity, \% } & \text { Specificity, \% } & \text { PPV, \% } & \text { NPV, \% }\end{array}$ Smoking per claims algorithm ${ }^{a}$ versus current smoker in chart ${ }^{b}$

$\begin{array}{lllll}\text { Algorithm 1 } & 4 & 20 & 1 & 191 \\ \text { Algorithm 2 } & 12 & 12 & 7 & 185 \\ \text { Algorithm 3 } & 15 & 9 & 14 & 178 \\ \text { Algorithm 4 } & 15 & 9 & 15 & 177\end{array}$

Smoking per claims algorithm ${ }^{a}$ versus current or former smoker in chart ${ }^{\mathrm{b}}$

\begin{tabular}{|c|c|c|c|c|c|c|c|c|}
\hline Algorithm 1 & 5 & 62 & 0 & 150 & 7.46 & 100.00 & 100.00 & 70.75 \\
\hline Algorithm 2 & 18 & 49 & 1 & 149 & 26.87 & 99.33 & 94.74 & 75.25 \\
\hline Algorithm 3 & 24 & 43 & 5 & 145 & 35.82 & 96.67 & 82.76 & 77.13 \\
\hline Algorithm 4 & 25 & 42 & 5 & 145 & 37.31 & 96.67 & 83.33 & 77.54 \\
\hline \multicolumn{9}{|c|}{ Dbesity per claims algorithm ${ }^{\mathrm{a}}$ versus $\mathrm{BMI} \geq 30$ in chart $^{\mathrm{b}}$} \\
\hline Algorithm 1 & 4 & 52 & 0 & 118 & 7.14 & 100.00 & 100.00 & 69.41 \\
\hline Algorithm 2 & 7 & 49 & 0 & 118 & 12.50 & 100.00 & 100.00 & 70.66 \\
\hline Algorithm 3 & 10 & 46 & 1 & 117 & 17.86 & 99.15 & 90.91 & 71.78 \\
\hline
\end{tabular}

$B M I$ body mass index, $P P V$ positive predictive value, NPV negative predictive value

a See Table 1 for description of each algorithm

${ }^{\mathrm{b}}$ Among those with non-missing information in the medical chart ( $n=216$ current smoker, $n=217$ current or former smoker, $\left.n=174 \mathrm{BMI}\right)$ 
of the range of opportunities to increase capture of comorbid conditions depending on the specific characteristics of the condition.

The inclusion of diagnosis codes from the mastectomy admission resulted in substantially increased prevalence of smoking, obesity, and hypertension. This finding is in agreement with previous studies which found the addition of diagnosis codes from the index admission was important to more accurately capture underlying comorbidities $[10,12,15]$. One explanation for this increase in prevalence could be the relevance of these conditions at the time of surgery. In the month prior to mastectomy in this younger population, $64 \%$ of the diagnoses coded on medical claims concerned the acute diagnosis (e.g., breast cancer, mammographic abnormality). Diagnoses of comorbid conditions may not have been considered relevant until the inpatient surgical admission, when hypertension, obesity, and smoking are important considerations in the administration of anesthesia and operative care. The inclusion of diagnoses from the mastectomy admission is justified for the comorbid conditions we chose to analyze since they are unlikely to arise during the mastectomy hospitalization.

There was a small but significant increase in the prevalence of hypertension and anemia when any two coded provider/outpatient facility claims were considered evidence for the condition, dropping the requirement for coding during encounters spaced at least 30 days apart. This may also be due to the younger mastectomy population we studied, in which healthcare encounters for workup and treatment are clustered during a relatively short time interval. We are unaware of any other studies that have assessed the spacing of outpatient claims. There was a large increase in prevalence of smoking and obesity when only one provider/outpatient facility claim was required to establish the diagnosis. The requirement of $\geq 2$ outpatient/provider claims to identify a condition was incorporated in comorbidity algorithms to account for inaccuracy in coding on outpatient claims [13, 24], and the lack of specific codes for diagnostic workup. Since no testing is required to establish the diagnosis of obesity or smoking, we removed the requirement for coding during multiple encounters, resulting in an 88 and $70 \%$ increase in the prevalence of obesity and smoking, respectively. The resulting increase put the calculated $12.65 \%$ smoking prevalence in our privately insured population much closer to the 2007 national estimate of current smoking in non-elderly adult women of $17.63 \%$.

Finally, the addition of outpatient prescription claims significantly increased the calculated prevalence of diabetes and hypertension, and in fact prescription claims identified more women with diabetes than medical claims. Medications have been used to identify chronic conditions alone [25-28], or in combination with diagnosis codes on medical claims [29, 30]. Adding medications to a medical claims algorithm improved identification of osteoporosis in a Canadian study [31, 32]. In contrast, prescription claims for smoking deterrents were uncommon and did not add to the prevalence estimate for smoking. Other investigators have used outpatient pharmacy data to estimate the prevalence of several chronic conditions, and found good agreement with health survey data for chronic conditions treated with specific agents, including diabetes and thyroid disorders [33]. The estimated prevalence of chronic conditions based on prescription drug utilization was higher than national estimates based on survey data in some studies [33, 34].

We found that the calculated prevalence for diabetes and hypertension in the mastectomy population were higher than prevalence estimates for women aged 18-64 years using the BRFSS and NHANES data. This could be due to the association of diabetes and hypertension with increased incidence of breast cancer [35-37]. Our calculated prevalence of anemia, which included iron deficiency and other anemias, may be higher than the national estimate since the NHANES testing was limited to iron deficiency anemia. In addition, the prevalence of anemia may be higher in the mastectomy population since some women may have developed anemia secondary to neoadjuvant chemotherapy, which could have been coded using a diagnosis code in our algorithm for "deficiency" anemia.

In contrast, our calculated prevalence of obesity was much lower than the estimated national prevalence of $26 \%$ in non-elderly adult women, likely due to the low sensitivity (18\%) of ICD-9-CM diagnosis codes to identify obesity that we found in medical record review. The very low sensitivity and high PPV of ICD-9-CM diagnosis codes to identify obesity in our study has also been reported for ICD-10 codes in Canada [38]. In our medical record validation we found that the median BMI for obese women with an ICD-9-CM diagnosis code for obesity was higher than the BMI of obese women who were not coded for obesity in the prior year, suggesting that morbid obesity is more likely to be coded than obesity per se. Supporting this is the similarity of the $6.31 \%$ prevalence of coded obesity in our mastectomy population to the $8.29 \%$ prevalence of morbid obesity in the NPCR Breast Cancer study [39].

Similarly, the prevalence of smoking in the mastectomy population defined by one or more encounters coded for tobacco use disorder was $12.87 \%$, lower than the 2007 national estimate of $17.63 \%$ from the BRFSS. Our lower prevalence may be due to the relatively low sensitivity $(62.50 \%)$ of coding for smoking we found compared to current smoking documented in the 
medical records. Coding of tobacco history or disorder had higher sensitivity to identify current smokers than ever smokers (62.50 \% vs. $37.31 \%$, respectively), but the PPV was lower for current compared to ever-smoking. These results suggest that tobacco use coding has relatively high specificity, but may not distinguish between current and former smokers.

For all comorbid conditions other than anemia, the prevalence estimates were higher when 12 diagnosis code fields were available in the later time period of the database rather than 5 fields. The trends of increasing prevalence with sequential changes in our coding algorithm were the same in both time periods. The reason for the lower prevalence of anemia in the later years of the study (2009-2011) may be due to the addition of a specific diagnosis code for antineoplastic chemotherapyinduced anemia in 2009, which is not included in the standard comorbidity category of anemia.

We found greatest improvement in our final algorithm in the younger third of the population compared with the older women in the cohort for diabetes, hypertension, and obesity. We hypothesized that comorbidities may not be captured as well among younger women with existing algorithms, owing to their different patterns of healthcare utilization compared to older women, which proved true for these three comorbid conditions. While improvements in capture of the five conditions we studied were evident in the older age groups as well (albeit to a lesser extent), particularly for anemia and smoking, these women in our cohort may be more similar to the older, sicker populations that were used to develop the traditional comorbidity algorithms $[1,4,13]$.

Previous studies have compared claims-based comorbidity measures or modifications based on improvement in ability to predict an outcome, rather than improved accuracy of condition identification. Most investigators have based improvement in comorbidity measures on improvement in model performance (e.g., concordance statistic) to predict mortality [7-10, 12, 14, 29, 40-43], although other outcomes such as healthcare costs [44, 45], readmissions [30], and progression of disease [41] have also been assessed. In contrast, we compared the prevalence of comorbidities calculated using claims data to health condition estimates in adult women of the same age reported in the BRFSS and NHANES survey populations. While we compared survey respondents with similar demographics to our population with respect to age, sex, and insurance status (BRFSS only), these national estimates may still not be completely comparable to the breast cancer population. The prevalence of a given comorbid condition in our commercially insured population may vary by other demographics that we could not account for, such as state of residence and type of insurance. In addition, the national estimates from the BRFSS survey are subject to limitations including self-report and recall bias.

We utilized a population of younger women undergoing mastectomy in order to assess modifications of a comorbidity algorithm in a non-elderly population. The patterns of healthcare utilization in our population of women, the majority of whom were newly diagnosed with breast cancer, may be different from other populations; therefore the generalizability of our findings is unknown. Future studies to determine if our findings hold in other non-elderly surgical and non-surgical populations, in men, and in patients with other types of health insurance are warranted. Men in particular have different patterns of healthcare utilization that may impact the performance of the algorithm.

\section{Conclusions}

We found progressively increasing prevalence of diabetes, hypertension, and anemia using claims data from a large population of non-elderly adult women undergoing mastectomy by altering the standard claims-based comorbidity algorithm to include diagnosis codes assigned during the surgical admission and removal of the requirement for spacing of diagnosis codes in outpatient encounters. Addition of outpatient pharmacy claims resulted in further increases in the calculated prevalence of diabetes and hypertension. We found progressively increasing prevalence of obesity and smoking by including diagnosis codes assigned during the surgical admission and by requiring only one coded inpatient or outpatient encounter, although medical record review to verify coding accuracy revealed low sensitivity of ICD-9-CM diagnosis codes to identify both health conditions. An important strength of our study is the large, longitudinal, population-based sample of younger persons representing hundreds of facilities from different geographic regions and different practice patterns. We assessed improvement in the coding algorithms compared to national survey estimates of the prevalence of the conditions in adult, non-elderly, insured women. Our results suggest a "one size fits all" approach to identifying comorbid conditions in claims data may not be the best way to identify individuals with a particular condition, and that investigators performing studies using claims data in younger, privately insured individuals may want to modify their strategy based on the particular comorbid conditions of interest. Additional research investigating strategies to optimize identification of comorbid health conditions in younger populations is needed to confirm these findings.

\section{Additional file}

Additional file 1: Diagnosis Codes and Drug Categories Used to Identify Comorbidities and Prevalence of Comorbidities Among Women From 2004-2008 vs. 2009-2011. (DOCX 15 kb) 


\section{Abbreviations}

BMl: Body Mass Index; BRFSS: Behavioral Risk Factor Surveillance System; Cl: Confidence Interval; CPT-4: Current Procedural Terminology, $4^{\text {th }}$ edition; HCPCS: Healthcare Common Procedure Coding System; HIRD ${ }^{\text {SM. HealthCore }}$ Integrated Research Database; ICD-9-CM: International Classification of Diseases, $9^{\text {th }}$ Revision, Clinical Modification; NHANES: National Health and Nutrition Examination Survey; NPV: Negative Predictive Value; PPV: Positive Predictive Value; UB-04: Uniform Billing Revenue

\section{Acknowledgments}

We thank Cherie Hill for database and computer management support.

\section{Funding}

Funding for this project was provided by the National Institutes of Health (NIH) (5R01CA149614 to MAO). MAO, DKW, and VJF were also supported in part by grant U54CK000162 from the Centers for Disease Control and Prevention (CDC) Epicenters Program (VJF). All aspects of this study including the findings and conclusions are those of the authors, who are responsible for its content, and do not necessarily represent the official view of the $\mathrm{NIH}$ or the CDC.

\section{Availability of data and materials}

The data for this study were acquired by the investigators at Washington University under a business agreement with Healthcore, Inc.. Investigators interested in acquiring the data should contact HealthCore, Inc. and reference this study.

\section{Authors' contributions}

KBN managed the data, conducted statistical analyses, and drafted the manuscript. AEW acquired and managed the data and critically reviewed the manuscript. KEB conducted the medical record review and critically reviewed the manuscript. DKW, DM, and VJF contributed to the study design and critically reviewed the manuscript. MAO conceived the study design, interpreted all findings, and drafted and revised the manuscript. All authors approved the final manuscript and agreed to be accountable for all aspects of the work.

\section{Competing interests}

DKW reports consultant work with Centene Corp., Worrell Inc., Cepheid Inc, Carefusion, and Pfizer Inc. for work outside the submitted manuscript. VJF reports personal fees from Battelle outside the submitted manuscript; her spouse is employed by Express Scripts. MAO reports consultant work with Pfizer, Merck, and Sanofi Pasteur and grant funding through Cubist Pharmaceuticals, Pfizer, and Sanofi Pasteur for work outside the submitted manuscript. All other authors report no conflicts of interest relevant to this article.

\section{Consent for publication}

Not applicable.

\section{Ethics approval and consent to participate}

This study was approved with a waiver of informed consent by the Human Research Protection Office at Washington University and by the Quorum Review IRB for the research activity at HealthCore.

\section{Author details}

'Division of Infectious Diseases, Department of Medicine, Washington University School of Medicine, 660 South Euclid Ave. Campus Box 8051, St. Louis, MO 63110, USA. ${ }^{2}$ HealthCore, Inc., 123 Justison St Suite 200,

Wilmington, DE 19801, USA. ${ }^{3}$ Division of Public Health Sciences, Department of Surgery, Washington University School of Medicine, 660 South Euclid Ave. Campus Box 8100, St. Louis, MO 63110, USA.

Received: 13 May 2015 Accepted: 4 August 2016

Published online: 16 August 2016

\section{References}

1. Charlson ME, Pompei P, Ales KL, MacKenzie CR. A new method of classifying prognostic comorbidity in longitudinal studies: development and validation. J Chronic Dis. 1987;40(5):373-83.

2. Deyo RA, Cherkin DC, Ciol MA. Adapting a clinical comorbidity index for use with ICD-9-CM administrative databases. J Clin Epidemiol. 1992;45:613-9.
3. Romano PS, Roos LL, Jollis JG. Adapting a clinical comorbidity index for use with ICD-9-CM administrative data: differing perspectives. J Clin Epidemiol. 1993;46(10):1075-9.

4. Elixhauser A, Steiner C, Harris R, Coffey RM. Comorbidity measures for use with administrative data. Med Care. 1998;36:8-27.

5. Agency for Healthcare Research and Quality. HCUP comorbidity software. Healthcare Cost and Utilization Project (HCUP). http://www.hcup-us.ahrq. gov/toolssoftware/comorbidity/comorbidity.jsp. Accessed 26 Sept 2014.

6. Sharabiani MT, Aylin P, Bottle A. Systematic review of comorbidity indices for administrative data. Med Care. 2012;50(12):1109-18.

7. Ghali WA, Hall RE, Rosen AK, Ash AS, Moskowitz MA. Searching for an improved clinical comorbidity index for use with ICD-9-CM administrative data. J Clin Epidemiol. 1996:49(3):273-8.

8. Southern DA, Quan H, Chali WA. Comparison of the Elixhauser and Charlson/Deyo methods of comorbidity measurement in administrative data. Med Care. 2004;42:355-60.

9. Piccirillo JF, Spitznagel Jr EL, Vermani N, Costas I, Schnitzler M. Comparison of comorbidity indices for patients with head and neck cancer. Med Care. 2004:42(5):482-6.

10. Baldwin LM, Klabunde CN, Green P, Barlow W, Wright G. In search of the perfect comorbidity measure for use with administrative claims data: does it exist? Med Care. 2006;44(8):745-53.

11. Needham DM, Scales DC, Laupacis A, Pronovost PJ. A systematic review of the Charlson comorbidity index using Canadian administrative databases: a perspective on risk adjustment in critical care research. J Crit Care. 2005;20(1):12-9.

12. Chu YT, Ng YY, Wu SC. Comparison of different comorbidity measures for use with administrative data in predicting short- and long-term mortality. BMC Health Serv Res. 2010;10:140.

13. Klabunde CN, Potosky AL, Legler JM, Warren JL. Development of a comorbidity index using physician claims data. J Clin Epidemiol. 2000:53:1258-67.

14. Wang PS, Walker A, Tsuang M, Orav EJ, Levin R, Avorn J. Strategies for improving comorbidity measures based on Medicare and Medicaid claims data. J Clin Epidemiol. 2000;53(6):571-8

15. Zhang JX, Iwashyna TJ, Christakis NA. The performance of different lookback periods and sources of information for Charlson comorbidity adjustment in Medicare claims. Med Care. 1999:37(11):1128-39.

16. Olsen MA, Nickel KB, Margenthaler JA, Wallace AE, Mines D, Miller JP, Fraser VJ, Warren DK. Increased risk of surgical site infection among breast-conserving surgery re-excisions. Ann Surg Oncol. 2015;22(6):2003-9.

17. Olsen MA, Nickel KB, Fox IK, Margenthaler JA, Ball KE, Mines D, Wallace AE, Fraser VJ. Incidence of surgical site infection following mastectomy with and without immediate reconstruction using private insurer claims data. Infect Control Hosp Epidemiol. 2015;36(8):907-14.

18. Sisco M, Du H, Warner JP, Howard MA, Winchester DP, Yao K. Have we expanded the equitable delivery of postmastectomy breast reconstruction in the new millennium? Evidence from the national cancer data base. J Am Coll Surg. 2012;215(5):658-66.

19. Lassman D, Hartman M, Washington B, Andrews K, Catlin A. US health spending trends by age and gender: selected years 2002-10. Health Aff (Millwood). 2014;33(5):815-22.

20. Green MA, Rowell JC. ICD-9-CM Coding. In: Understanding health insurance: a guide to billing and reimbursement. 11th ed. Clifton Park: Delmar, Cengage Learning; 2013. p. 143-200.

21. Centers for Disease Control and Prevention (CDC). Behavioral Risk Factor Surveillance System Survey Data. U S Department of Health and Human Services. http://www.cdc.gov/brfss/annual_data/annual_data.htm. Accessed 29 July 2014.

22. From the Centers for Disease Control and Prevention. Iron deficiencyUnited States, 1999-2000. JAMA. 2002:288(17):2114-6.

23. Wolin KY, Carson K, Colditz GA. Obesity and cancer. Oncologist. 2010;15(6):556-65.

24. Quam L, Ellis LB, Venus P, Clouse J, Taylor CG, Leatherman S. Using claims data for epidemiologic research. The concordance of claims-based criteria with the medical record and patient survey for identifying a hypertensive population. Med Care. 1993:31(6):498-507.

25. Von Korff M, Wagner EH, Saunders K. A chronic disease score from automated pharmacy data. J Clin Epidemiol. 1992:45(2):197-203.

26. Clark DO, Von KM, Saunders K, Baluch WM, Simon GE. A chronic disease score with empirically derived weights. Med Care. 1995:33(8):783-95.

27. Fishman PA, Goodman MJ, Hornbrook MC, Meenan RT, Bachman DJ, O'Keeffe Rosetti MC. Risk adjustment using automated ambulatory pharmacy data: the RxRisk model. Med Care. 2003;41(1):84-99. 
28. Halfon P, Eggli Y, Decollogny A, Seker E. Disease identification based on ambulatory drugs dispensation and in-hospital ICD-10 diagnoses: a comparison. BMC Health Serv Res. 2013;13:453.

29. Schneeweiss S, Seeger JD, Maclure M, Wang PS, Avorn J, Glynn RJ. Performance of comorbidity scores to control for confounding in epidemiologic studies using claims data. Am J Epidemiol. 2001;154(9):854-64.

30. Parker JP, McCombs JS, Graddy EA. Can pharmacy data improve prediction of hospital outcomes? Comparisons with a diagnosis-based comorbidity measure. Med Care. 2003:41(3):407-19.

31. Lix LM, Yogendran MS, Leslie WD, Shaw SY, Baumgartner R, Bowman C, Metge C, Gumel A, Hux J, James RC. Using multiple data features improved the validity of osteoporosis case ascertainment from administrative databases. J Clin Epidemiol. 2008:61(12):1250-60.

32. Hudson M, Avina-Zubieta A, Lacaille D, Bernatsky S, Lix L, Jean S. The validity of administrative data to identify hip fractures is high-a systematic review. J Clin Epidemiol. 2013;66(3):278-85.

33. Chini F, Pezzotti P, Orzella L, Borgia P, Guasticchi G. Can we use the pharmacy data to estimate the prevalence of chronic conditions? a comparison of multiple data sources. BMC Public Health. 2011;11:688.

34. Orueta JF, Nuno-Solinis R, Mateos M, Vergara I, Grandes G, Esnaola S. Monitoring the prevalence of chronic conditions: which data should we use? BMC Health Serv Res. 2012:12:365.

35. Largent JA, Bernstein L, Horn-Ross PL, Marshall SF, Neuhausen S, Reynolds P, Ursin G, Zell JA, Ziogas A, Anton-Culver H. Hypertension, antihypertensive medication use, and breast cancer risk in the California Teachers Study cohort. Cancer Causes Control. 2010;21(10):1615-24.

36. Michels KB, Solomon CG, Hu FB, Rosner BA, Hankinson SE, Colditz GA, Manson JE. Type 2 diabetes and subsequent incidence of breast cancer in the Nurses' Health Study. Diabetes Care. 2003;26(6):1752-8.

37. Sabatino SA, Thompson TD, Wu XC, Fleming ST, Kimmick GG, TrenthamDietz A, Cress R, Anderson RT. The influence of diabetes severity on receipt of guideline-concordant treatment for breast cancer. Breast Cancer Res Treat. 2014:146(1):199-209.

38. Martin BJ, Chen G, Graham M, Quan H. Coding of obesity in administrative hospital discharge abstract data: accuracy and impact for future research studies. BMC Health Serv Res. 2014;14:70.

39. Kimmick G, Fleming ST, Sabatino SA, Wu XC, Hwang W, Wilson JF, Lund MJ, Cress R, Anderson RT. Comorbidity burden and guideline-concordant care for breast cancer. J Am Geriatr Soc. 2014;62(3):482-8

40. Klabunde CN, Legler JM, Warren JL, Baldwin LM, Schrag D. A refined comorbidity measurement algorithm for claims-based studies of breast, prostate, colorectal, and lung cancer patients. Ann Epidemiol. 2007;17(8):584-90.

41. Lix LM, Quail J, Fadahunsi O, Teare GF. Predictive performance of comorbidity measures in administrative databases for diabetes cohorts. BMC Health Serv Res. 2013;13:340.

42. Sarfati D, Gurney J, Stanley J, Lim BT, McSherry C. Development of a pharmacy-based comorbidity index for patients with cancer. Med Care. 2014;52(7):586-93

43. Schneeweiss S, Wang PS, Avorn J, Glynn RJ. Improved comorbidity adjustment for predicting mortality in Medicare populations. Health Serv Res. 2003;38(4):1103-20.

44. Farley JF, Harley CR, Devine JW. A comparison of comorbidity measurements to predict healthcare expenditures. Am J Manag Care. 2006;12(2):110-9.

45. Ou HT, Mukherjee B, Erickson SR, Piette JD, Bagozzi RP, Balkrishnan R. Comparative performance of comorbidity indices in predicting health carerelated behaviors and outcomes among Medicaid enrollees with type 2 diabetes. Popul Health Manag. 2012;15(4):220-9.

\section{Submit your next manuscript to BioMed Central and we will help you at every step:}

- We accept pre-submission inquiries

- Our selector tool helps you to find the most relevant journal

- We provide round the clock customer support

- Convenient online submission

- Thorough peer review

- Inclusion in PubMed and all major indexing services

- Maximum visibility for your research

Submit your manuscript at www.biomedcentral.com/submit
Biomed Central 